\title{
MaLtRato INFANTIL: RELEVAMIENTO EPIDEMIOLOGICO EN LA POBLACION ESCOLARI- ZADA DE LA CIUDAD AUTONOMA DE BUENOS AIRES
}

\author{
CHILD ABUSE: EPIDEMIOLOGICAL DATA OF ENROLLED \\ IN SCHOOL POPULATION BUENOS AIRES CITY
}

\author{
Prof. Dra. María Inés Bringiotti* \\ Universidad de Buenos Aires, Argentina
}

\begin{abstract}
Resumen: Esta investigación replica la realizada en las escuelas dependientes del Gobierno de la Ciudad de Buenos Aires -nivel inicial y nivel primario- hace 10 años. Se releva el número de niños/niñas que pudieran estar sufriendo alguna forma de malos tratos intrafamiliares, así como formas de violencia entre pares y entre grupos en el ámbito escolar. Se analiza su relación con edad, sexo, tipo de maltrato/violencia, factores de riesgo, problemas de aprendizaje y conducta, y porcentaje de los mismos que reciben atención. La comparación entre ambos estudios determina un $36 \%$ de aumento en la detección de casos, las razones deben ser analizadas cuidadosamente ya que inciden tanto la mayor capacitación para visualizarlos, como el aumento de conductas violentas en el actual contexto sociocultural.
\end{abstract}

Palabras claves: Maltrato a los niños; Epidemiología; Factores de Riesgo; Prevención

\begin{abstract}
The present study replicates a previous one done 10 years ago at primary public schools of Buenos Aires City. Data was obtained about children who might be suffering any kind of abuse in their family or violence between peers or groups in school settings. Analyze was made between abuse and age, gender, kind of abuse, risk factors, learning and behavior problems, and rate of children in treatment. 1590 cases were detected, for a $36 \%$ increase in detection rate. Careful evaluation must be made because this raise might be related with training in detection as well as increasing violence in social and cultural context.
\end{abstract}

Keywords: Child abuse; Epidemiology; Risk factors; Prevention

\section{INTRODUCCIÓN}

En las últimas décadas de nuestra antigua civilización ha comenzado a descorrerse un telón de fondo sobre las situaciones relacionadas a la problemática de la violencia familiar.

Hoy hablamos de un conjunto de situaciones violentas que ocurren en su seno: violencia conyugal y/o violencia cruzada en la pareja, mal-trato infantil, maltrato a los ancianos, maltrato de los hijos hacia los padres y violencia en el noviazgo.

El maltrato aplicado a los niños se observa desde los inicios de la historia, una recorrida por los autores que analizan la historia de la infancia registra una amplia gama de acciones $u$ omisiones cometidas en perjuicio de los mismos (Aries, 1987; Badinter, 1991; De Mausse, 1974). El abandono y los malos tratos eran prácticas comunes en la educación de los niños (Burguière, Klapisch-Zuber, Segalen \& Zonabend, 1988). La naturalización del castigo en las prácticas educativas y el abuso sexual llevado a cabo en diferentes sectores, ocultó durante años la crudeza de la situación. En 1964,

* El Equipo de trabajo lo constituyen: Lic. M.E. Asnicar; Coordinadora de Trabajo de Campo. Lic. S. Abad; Lic. L. Balduzzi; Lic. G. Lamela; Lic. L. Mígueles; Lic. S. Rochel Cabo; Dra. G. Santamaría; Entrevistadoras. Lic. A., Aneiros; Lic. M. E., Origliano; Lic. S., Crusciani; Lic. J., Fulugulio; Lic. M., Hernández; Lic. M., Morán; Lic. C., Ríos; Lic. G., Vázquez; Pasantes del Nivel Superior de Ciencias de la Educaciòn - UBA-.

Correspondencia: Dra. María Inés Bringiotti, Profesora de la Facultad de Filosofía y Letras, Universidad de Buenos Aires; Programa de Investigación en Infancia Maltratada. Correo Electrónico: mibringiotti@fibertel.com.a 
Kempe -médico pediatra forense americanologra en la Convención Anual de Pediatría que sea aceptado por primera vez, el síndrome del niño apaleado. Desde el siglo XIX los médicos forenses registraban casos que despertaban sospechas, pero que eran calificados como accidentes domésticos. A partir de allí, cuando se comienza a hablar de maltrato infantil, aunque referido casi exclusivamente al maltrato físico.

¿De qué se habla cuando nos referimos a maltrato infantil intrafamiliar?. Una definición amplia y consensuada a nivel internacional considera al maltrato infantil como:

"Cualquier daño físico o psicológico no accidental contra un menor de 16 ó 18 años -según el régimen de cada país-, ocasionado por sus padres o cuidadores que ocurre como resultado de acciones físicas, sexuales o emocionales de omisión o comisión y que amenazan el desarrollo normal tanto físico como psicológico del niño/a" (y podríamos agregar social); (Gracia Fuster \& Musitu Ochoa, 1993, p.35).

La primera tipología del maltrato infantil (década de 1965 a 1975) incluye cinco subtipos básicos: maltrato físico, abandono físico, maltrato emocional, abandono emocional y abuso sexual. Posteriormente se han ido agregando, en los últimos 25 años, otras formas que ocurren en el seno de familias disfuncionales: maltrato prenatal, explotación laboral, mendicidad, corrupción, prostitución infantil, síndrome de Mun-chaussen, falta de control parental sobre la conducta de los hija/os, adopción inadecuada. Finkelhor (1990), citado por Bringiotti (2000), especialista americano en abuso sexual infantil, incluye como forma de maltrato infantil -institucional y social-al secuestro y sustitución de identidad, mencionando los casos ocurridos en Argentina y Guatemala.

Dentro de los estudios explicativos encarados para dar cuenta del porqué de la ocurrencia del maltrato encontramos los modelos de primera generación o unicausales (1970), que ponían el acento en: a) variables psicológicas de la personalidad del sujeto maltratador -modelo psicológico psiquiátrico-, b) variables de corte socioeconómico -modelo socio-lógico-y c) las características del niño -modelo de vulnerabilidad del niño-. Más tarde, en la década de los 80 surgen los modelos de segunda generación o de interacción social, entre ellos, el clásico ecológico ecosistémico, que analiza el conjunto de factores de riesgo y compensatorios asociados a la ocurrencia de los malos tratos (Belsky,
1980; Cichetti \& Rizley, 1981). Estos modelos buscaban articular el conjunto de variables detectadas desde cada línea de abordaje de los modelos de la primera generación e incluir el impacto de las mismas entre sí. Posteriormente en la década de los 90 surgen los modelos de la tercera generación o modelos cognitivos: procesamiento de la información (Milner, 1993) y afrontamiento al estrés (Hillson y Kuiper, 1994, citado por Cantón Duarte \& Cortés Arboleda, 1997). Estos Modelos analizan la conformación de las cogniciones que llevan a las respuestas violentas.

El modelo ecológico ecosistémico, actualmente el más conocido y utilizado, presenta un conjunto de variables que, articuladas entre sí, potencian el riesgo de ocurrencia del maltrato infantil, señalando que tal asociación no debe ser entendida en ningún momento como de causalidad o determinista. La ventaja del mismo ha sido incluir en un modelo articulado un conjunto de factores psicológicos y sociales asociados a la ocurrencia de los malos tratos infantiles. El objetivo era superar las posturas extremas que adjudican la responsabilidad, por un lado a los individuos -padre, madre, cuidadores-definidos primordialmente como sujetos psíquicos o, por el otro, a las deficientes condiciones socioe-conómicas entendidas como genera-doras del maltrato, independiente de las estructuras de personalidad y la historia de sus miembros. Los mo-delos teóricos lo abordan como resol-tados de la interrelación entre lo indi-vidual, lo familiar, lo social y lo cultural, con un criterio amplio de análisis.

A nivel sociológico se señalan como aspectos influyentes: desocupación, insatisfacción laboral, niveles socioeconómico y educativo bajos, aislamiento social, déficit de soporte social, zonas de residencia de alto riesgo, condiciones de vivienda inadecuadas, familias mono-parentales, y alto número de hijos con inadecuado espacio intergenésico, entre los más destacados.

En el plano psicológico encontramos: altos niveles de ansiedad y depresión, irritabilidad, falta de recursos para el enfrentamiento de problemas, impulsividad, historia de malos tratos en la propia infancia de los padres, edad en que la madre tiene a su primer hijo, déficit en la c-pacidad empática, inadecuado manejo del estrés, e inhabilidades parentales para la educación de los hijos.

El aspecto cultural aporta elementos referi- 
dos a las características de cada contexto, en lo relativo a los patrones tolerados de castigos y sanciones; valores y actitudes hacia la infancia y hacia los roles genéricos; aprobación del uso de la violencia en la educación y en las relaciones entre pares.

Respecto a las características del niño, se han incluido un conjunto de situaciones detectadas en clínica neonatal temprana como de riesgo para el maltrato y/o abandono infantil: hijos no deseados, nacimiento prematuro, bajo peso, problemas en el sueño y la alimentación, handicaps físicos o psíquicos, niños producto de relaciones incestuosas, y niños nacidos después de la separación de los padres, entre las más importantes.

La aplicación del modelo eco-lógico ecosistémico (Belsky, 1993) en los abordajes investigativos y terapéuticos resultó inicialmente de mucha utilidad para determinar los factores de riesgo presentes en familias/contextos maltratantes o de alto riesgo. Sin embargo, la experiencia mostró que frente a la presencia de factores similares las respuestas eran diferentes, así, la investigación en el tema permitió incorporar los factores compensatorios y recientemente el concepto de resiliencia (Barudy Labrin, 2006)

Los cambios estructurales ocurridos en los últimos años en nuestro país son el reflejo de crisis compartidas principalmente por los países latinoamericanos, pero que también se reflejan a nivel mundial en las condiciones socioeconómicas cotidianas de vida de las familias. A partir de los 80 se van gestando cambios que impactan al interior de las mismas. La influencia de diversos factores como el aumento del embarazo adolescente o madres jóvenes sin experiencia, de familias monoparentales generalmente con jefatura femenina, de problemas económicos para el sostenimiento del hogar, del debilitamiento de los lazos sociales, del estrés producido por el conjunto de situaciones anteriores, se ve reflejada en la calidad de vida familiar y concretamente en los niños en su acceso a la salud y la educación y en la ausencia de modelos parentales «saludables».

$\mathrm{Al}$ acentuarse los problemas socioeonómicos y estructurales, también aumentaron las violencias ejercidas y sufridas desde diferentes sectores - individual, familiar, institucional y social. La violencia social sufrida por estos sectores se traslada a una violencia que ocurre en el ámbito privado/familiar y se extiende a los diferentes miembros -la mujer, la pareja entre sí, los niños, los ancianos-, conformando la ins-talación de un ciclo de la violencia que de no mediar algún tipo de intervención, tendrá altas posibilidades de repetirse con los futuros hijos - nos referimos a la llamada transmisión generacional del maltrato- (De Paúl, 1987). La violencia social y la violencia familiar, mantienen al menor en una situación básica de carencia y desprotección, que lo torna vulnerable a la repetición del circuito de maltrato y marginalidad - numerosas investigaciones han mostrado la asociación entre ambas situaciones - (Cantón Duarte \& Cortés Arboleda, 1997). La capacidad de la familia para adaptarse a las perturbaciones provenientes del medio exterior dependerá de la magnitud y calidad de las perturbaciones, pero además de la situación de la familia en el momento de ser afectada. En algunas situaciones, las familias agotadas por las presiones del contexto, se encuentran imposibilitadas para producir modificaciones y respuestas adecuadas, éste es el caso de aquellas familias donde se produce violencia familiar y maltrato a los niño/as en un contexto de crisis. (Barudy Labrin, 1998).

\section{ANTECEDENTES}

Desde el Programa de Investigación en Infancia Maltratada que funciona en la Universidad de Buenos Aires, nuestro equipo llevó a cabo en las escuelas, un relevamiento epidemiológico para detectar los casos de niños/ as que estuvieran sufriendo alguna forma de malos tratos intrafamiliares. El mismo se extendió entre 1992 y 1993 al partido de Avellaneda -Gran Buenos Aires- y entre 1994/1996 a las escuelas dependientes de la Secretaría de Educación-Capital Federal-, con el fin de detectar la mag-nitud del problema a través del ámbito educativo. Así se obtuvo una evaluación precisa del alcance de la problemática del maltrato infantil, sus diferentes tipologías, factores de riesgo y zonas de alto riesgo.

Pasada una década de tal relevamiento se plantea un estudio similar a fin de comparar los cambios en la cantidad de casos, tipos y factores de riesgo, ya que en general se suele carecer de estudios sistemáticos de tipo longitudinal que faciliten el análisis y la elaboración de programas de prevención e intervención. Este segundo estudio, debido a problemas de tiempo y pre-supuesto, se redujo a las escuelas dependientes de la Secretaría de Educación 
-nivel inicial y primario en los años 2004/05 $y$, las escuelas especiales en el año 2006(UBACYT, F144). Por lo tanto, la información recabada corresponde al ámbito capitalino, y exclusivamente a las escuelas dependientes de la Secretaría de Educación de la Ciudad Autónoma de Buenos Aires.

\section{MATERIAL Y MÉTODO}

\section{Objetivos de la investigación}

1. Conocer el número de casos de menores maltratados concurrentes a las escuelas dependientes de la Secretaría de Educación de la Ciudad Autónoma de Buenos Aires -nivel inicial y primario- a través de una muestra de distritos y escuelas.

2. Discriminar en los casos detectados los de evidencia de los de sospecha.

3. Determinar los factores de riesgo asociados a la ocurrencia de los mismos.

4. Relacionar los casos detectados con el sexo y la edad del niño/a, grado o sección, distrito escolar y tipo de maltrato.

5. Conocer el número de casos que presentan dificultades en la escolarización, como atraso o repi-tencia.

6. Determinar el porcentaje de los mismos que se encuentran bajo alguna forma de atención /tratamiento.

\section{Muestra}

En Capital Federal funcionan 21 Distritos Escolares, cada uno de los cuales incluye entre 18 a 22 escuelas de nivel inicial y otro tanto de nivel primario. Para toda la Capital el número de instituciones educativas llega a 500 para cada nivel. El nivel inicial es obligatorio a partir de los 4 años, concurriendo a salitas de 4 y 5 años; sin embargo hay guarderías maternales que reciben niños más pequeños -de 2 y 3 años-. El nivel primario abarca de 6 a 12/13 años. Una muestra representativa de los niveles inicial y primario debía abarcar un porcentaje mayor al $20 \%$. Se sortearon, entonces, 8 distritos y 1 de reemplazo ante cualquier even-tualidad. En cada uno de ellos se sortearon 4 escuelas de nivel inicial y 4 de nivel primario con sus reemplazos. Las 32 instituciones sorteadas eran representativas de los niveles inicial y primario, y de la totalidad de turnos y grados/secciones.

Los distritos y las escuelas seleccionadas, fueron sorteados por azar teniendo todos, las mismas posibilidades de ser elegidos. El sorteo fue realizado por nuestro equipo ante la presencia de los directivos de los niveles inicial y primario en la sede de la Secretaría de Educación.

En algunos casos, no fue posible relevar la totalidad de grados/ secciones por cambio de los do-centes, por problemas coyunturales de la institución o por la negativa de la escuela a participar en ese momento. La muestra seleccionada cubría el $38 \%$ del total de los distritos escolares y el $7 \%$ del total de escuelas de nivel inicial y primario, porcentajes considerados adecuados por el estadístico consultado.

\section{Instrumento utilizado}

\section{Cartilla para docentes}

Cada una de las escuelas sorteadas fue relevada en la totalidad de grados primarios y secciones de jardín. Para ello se utilizó la modalidad empleada en el relevamiento anterior, previamente se realizaba una reunión explicativa, y luego se entregaba a cada docente una cartilla que debía responder en forma anónima y en la cual los casos detectados también debían ser anónimos a fin de asegurar la confiabilidad de las respuestas.

La misma fue adaptada a nuestro contexto a partir de la utilizada en la Investigación Epidemiológica realizada en España, País Vasco en 1990. (De Paúl, comunicación per-sonal, 1990). El trabajo realizado anteriormente en Avellaneda, y Capital Federal, nos permitió evaluar la cartilla utilizada y realizar los ajustes necesarios. Nuestro modelo también fue adaptado y aplicado en Sarmiento, localidad a 120 $\mathrm{km}$ de Comodoro Rivadavia, Chubut (Intebi, 2006) y en Riberao Preto, Sao Paulo, Brasil, (Matias, 2004), resultando adecuado para los fines planteados.

En la cartilla se presentaba una tipología del maltrato infantil con definiciones explícitas para cada uno de los tipos incluidos en la investigación: maltrato físico, abandono físico, maltrato emocional, abandono emocional, abuso sexual, trabajo del menor, mendicidad, corrupción, participación del menor en acciones delictivas junto con su familia y falta de control parental sobre la conducta de sus hijos, y sus correspondientes indicadores. Ello tenía el objetivo de orientar a los docentes sobre los casos a detectar y podría también brindar información suplementaria sobre el problema. Esas hojas podían ser desglosadas por el docente para ser 
utilizadas como material de consulta.

En el actual relevamiento se incluyeron otras formas como violencia entre niños y violencia entre grupos. Otra modificación fue el desglose de las categorías abandono físico, trabajo del menor y mendicidad, en los tipos familiar y social (i. e. abandono físico familiar y abandono físico social) a fin de registrar adecuadamente los casos en los cuales la familia tiene inhabilidades en la crianza y mayor respon-sabilidad, de aquellos otros casos - cada vez más numerosos- que son producto de la aguda crisis socioeconómica de los últimos años.

Posteriormente se presentaban los cuadros a completar por el docente: el cuadro 1 donde debían registrar en forma anónima el total de alumnos, que en su grado o sección, presentaban alguna de las formas de maltrato mencionadas, especificando únicamente el sexo y la edad y sobre los cuales tuviera la certeza o evidencia de que el maltrato ocurría.

Otro cuadro similar -cuadro 2 en el material entregado a los docentes-indicaba los casos de coocurrencia de más de un tipo de maltrato en el mismo menor. Los dos cuadros mencionados se referían a casos de evidencia. Otros dos cuadros -cuadros 3 y 4- permitían el volcado de los casos de sospecha -de un sólo tipo de maltrato o de coocurrencia de más de un tipo-.

Por último, se presentaba el cuadro 5 con un listado de factores de riesgo asociados al maltrato infantil, extraídos del modelo ecológico ecosistémico, para constatar su ocurrencia y el peso que adquieren en nuestro contexto socioeconómico y cultural. A partir del relevamiento anterior y teniendo en cuenta los cambios ocurridos en los últimos años en nuestro país, especialmente luego de la crisis de diciembre de 2001, se ajustaron los factores mencionados para su aplicación en esta in-vestigación. La cartilla fue aplicada entre los meses de agosto a noviembre de 2004 y en el transcurso del año escolar del 2005. Pos-teriormente se realizó el relevamiento en las escuelas especiales.

\section{PRINCIPALES RESULTADOS}

Las secciones y grados relevados cubren una matrícula de 42.000 alumnos aproximadamente, sobre los cuales han respondido entregando la cartilla -con o sin casos-docentes que cubren una matrícula de 32.000 alumnos, es decir, el $76 \%$ de la muestra. En el caso del $24 \%$ de los alumnos restantes, los docentes no han devuelto la cartilla, pese a sucesivos pedidos y reclamos, o bien se trata de escuelas que decidieron no participar del relevamiento.

Respecto al total de casos por nivel de escolarización, en la muestra del nivel inicial se han detectado 265 casos y en el primario 1325 casos de malos tratos, sumando un total de 1590 niños/niñas. Si tenemos en cuenta que el número de instituciones escolares es casi similar -398 y 420 respectivamente para los niveles inicial y primario-el número de casos detectados en el nivel primario supera marcadamente al inicial, aunque este último ha aumentado en relación al relevamiento anterior su proporción respecto a primaria. Una década atrás se detectaron 126 y 1039 casos respectivamente (1994/1997), en este caso el nivel inicial cubría un $12 \%$ respecto al número de casos de primaria y ahora ascendió al $20 \%$ respecto a la misma.

En cuanto al número total de casos registrados, pasaron de 1165 a 1590, con un aumento del $36 \%$ de registros.

Los casos registrados por los docentes fueron discriminados según tuvieran evidenciasospecha de su ocurrencia: en este segundo releva-miento pudimos observar mayores temores y cuidados a la hora de responder. El porcentaje de evidencia -en el nivel primario- bajó del $80 \%$ (estudio anterior) al $69.3 \%$, mos-rando de todas formas el cono-cimiento que poseen los docentes de la ocurrencia del mismo, siendo agentes fundamentales a la hora de la detección. En el 30.2\% restante se posee sospecha acerca de la presencia de malos tratos a los niños. El porcentaje de casos de evidencia en el nivel inicial -70.6\%-es levemente mayor que el promedio para el total de la muestra. Podría tratarse de una mejor detección debida a una mayor capa-citación por parte de los docentes al tratarse de niños más pequeños.

En cuanto al sexo del niño/a en el nivel primario, se ha detectado un $63 \%$ de maltrato en varones, mientras que anteriormente era de $54.8 \%$. Esta característica fue observada en estudios epidemiológicos realizados en otros países y en los realizados anteriormente por nuestro equipo, los cuales mostraron que los varones son mayoritariamente los detectados o los que sufren formas de maltrato infantil más visibles. En el caso de las nenas la disminución al 33\% merece un análisis más profundo. Muchas formas de abuso sexual intrafamiliar -como exhibicionismo, toqueteos, caricias y otras modalidades de abuso sexual sin pene- 
tración- tienen como víctimas principalmente a las niñas y esta situación se ampara en lo que ocurre en el ámbito privado y no se registra oficialmente. Algo similar ocurre con formas exageradas de colaboración en lo doméstico por parte de las niñas -cuidado de hermanos menores, cocinar, lavar, salir a hacer los mandados- donde la distribución social de los roles y su consiguiente aceptación hace también invisible esta situación.

En el nivel inicial, el 3.5\% de los casos se detectaron en niños de 2 años; el 19\% en los de 3 años; el $47 \%$ en los de 4 años y el $30.5 \%$ restante en los de 5 años. Las edades presentadas coinciden a las que se registran en las secciones del jardín, observándose una correspondencia entre sala y edad.

Respecto a las características del maltrato detectado, se presenta en primer lugar la categorización de ocurrencia de malos tratos para el total de casos detectados en el nivel primario, según los cuatro tipos de ocurrencia.

- Evidencia 1 solo tipo (n: 772 ) - 48\% (antes $55.5 \%$ )

- Evidencia más de un tipo (n: 298) - 19\% (antes 24\%)

- Sospecha 1 solo tipo (n: 420 ) - 26\% (antes 15\%)

- Sospecha más de un tipo (n: 100) - 7\% (antes 5\%)

En el nivel inicial la distribución de casos es la siguiente:

- Evidencia 1 solo tipo (n: 161) - 60\% (antes $55.5 \%$ )

- Evidencia más de un tipo (n: 20) - 8\% (antes 24\%)

- Sospecha 1 solo tipo (n: 55) - 21\% (antes 15\%)

- Sospecha más de un tipo (n: 29) - 11\% (antes 5\%)

En cuanto a las formas más habituales de malos tratos, a continuación se presentan los porcentajes obtenidos en este relevamiento:

Comparando ambos estudios, en el período anterior se registraron 650 casos de evidencia de un solo tipo, en éste 720 casos. En este relevamiento se incluyeron las formas de violencia entre niños y entre grupos, lo que modifica los valores relativos. Para poder comparar los
Volenda entre pares N: 282 - 36\%

Abandono figico familiar $\mathrm{N}: 106=14 \%$.

Abardano amoganal N $82-11 \%$

Faita dē contrôl parental W: 64 - 8\%.

Maltrato fistoo $\mathrm{N}: 56=7 \%$

Abandono fisico gocial N: $50=7 \%$.

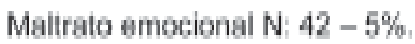

Vobencia entre ğrupos $\mathbf{N}: 25-3 \%$.

Violengia institudonal N: $24-3 \%$.

Abugo sexual $12-1.7 \%$

Trabajo del mencr familiar Na $012 \%$

Comupoion $\mathrm{N}: 8-1,1 \%$.

Mondididad soblal N: 7 = 1\%

Açiones dẹlictivas N: $7-1 \%$

resultados, separamos los casos de violencia entre niños y entre grupos y analizamos los otros tipos de malos tratos.

Ello nos permitió observar que el abandono físico, sumando el abandono físico familiar y el social, asciende al $36 \%$ de los casos de un solo tipo con evidencia, aumentando respecto al estudio anterior, de un $32 \%$.

Han aumentado también el abandono emocional, la falta de control parental, el maltrato emocional, el abuso sexual, la corrupción y la mendicidad, disminuyendo el porcentaje de mal-trato físico y trabajo del menor-esto debe tomarse como el efecto de una redistribución de los tipos, la realidad muestra que ambos ocurren en proporciones muy elevadas, sólo que al mantenerse estables o con un aumento no tan significativo "aparecen" como menores frente a la visibilidad de las otras formas de malos tratos-.

Es positivo el registro de casos cuyos tipos exceden el maltrato físico, ya que tradicionalmente el maltrato infantil suele ser reducido a las formas de castigo físico; en estos momentos ha 


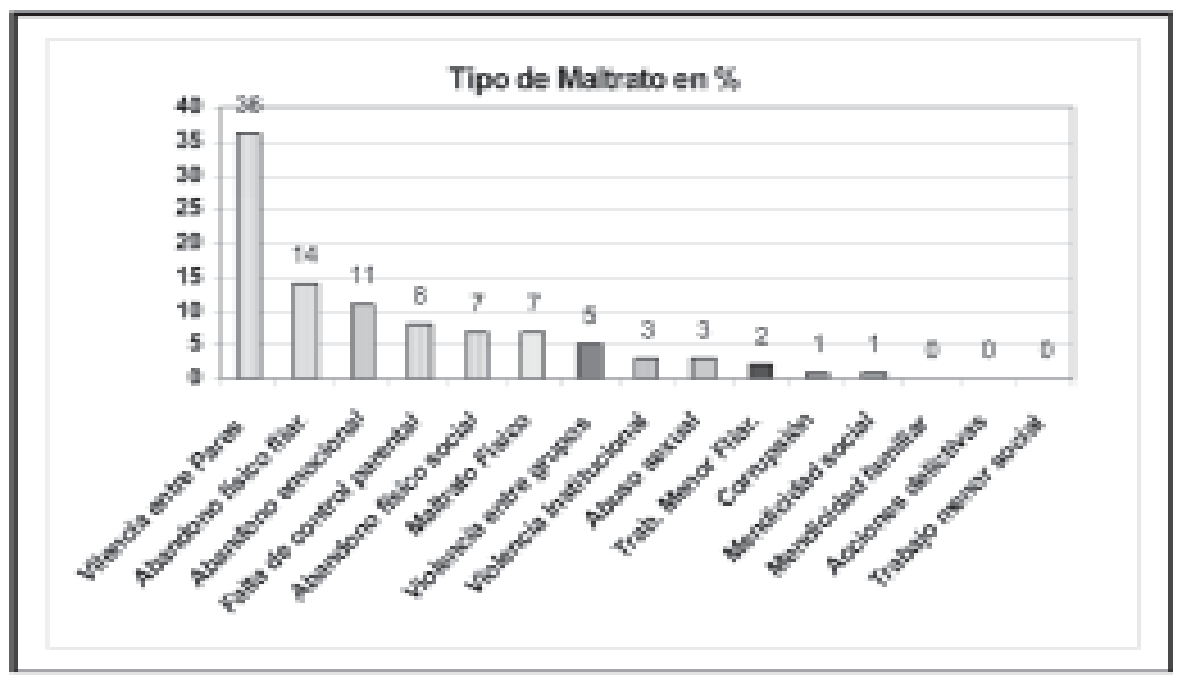

Cuadro 1: THPOS DE BLALOS TFATOS

aumentado el conocimiento y se han visibilizado otras formas igualmente dañinas.

Respecto al impacto o los efectos del maltrato, observemos que en los 29 jardines relevados y las 33 escuelas primarias, se detectaron 1590 casos de chicos maltratados, con una ocurrencia de 2563 situaciones de malos tratos, si tomamos en cuenta los casos en que el mismo chico sufre más de un tipo de maltrato. Esto nos señala una ocurrencia de 1.6 formas de maltrato por chico para el total de la muestra -inicial y primaria.-

Para el total de la muestra: 1.6 TIPO DE MALTRATO POR CHICO.

PARA EL TOtAL DEL NIVEL INICIAL: 1.4.

PARA EL TOTAL DEL NIVEL PRIMARIO: 1.8.

El análisis de los factores de riesgo, asociados a la problemática del maltrato infantil, se realizó con la misma metodología utilizada en los relevamientos anteriores. En primer lugar se extrajeron los puntajes absolutos que mostraban el valor puntual de cada factor -el $47 \%$ de las familias presentaban Problemas económicos graves-, el 43\% Desempleo y así sucesivamente. Por otra parte se extrajeron los puntajes relativos con ba-se 100\% -15\% Problemas económicos graves, 13\% Desempleo.. -

Entre los factores más relevantes asociados al maltrato se encuentran aquellos relacionados a las problemáticas socioeconómicas -desem-pleo, problemas económicos graves o moderados, bajo nivel cultural de los padres, que abarcan un $46.5 \%$-, y los específicos de las disfunciones familiares -familia monoparental, separación o divorcio y problemas conyugales-, que suman un $22.5 \%$.

Hemos incorporado dentro de los factores de riesgo, aspectos relacionados a la violencia, como violencia conyugal y maltrato en la infancia de los padres, donde a pesar de tratarse de temas que podían ser ignorados por los maestros, cubren el $9 \%$ de los casos, tratándose de situaciones que comienzan a mostrarse y registrarse.

Casi un $11 \%$ de los casos presentan alguna forma de enfermedad física o psíquica -enfermedad física prolongada, retraso mental, ansiedad, depresión, alcoholismo o drogadicción-. Estos dos últimos fueron registrados en el $6 \%$ de las familias.

Resulta necesario aclarar que los datos presentados se refieren aproximadamente al $75 \%$ de las familias de los casos detectados, ya que para el $25 \%$ restante, la respuesta es "no sabe/ no contesta". Los factores detectados indican un mejor conocimiento de las características del niño y su familia, en el nivel inicial que en el nivel primario.

Posteriormente se analizó la presencia de dificultades como atraso o repitencia, para incluir la repetición de grados o el atraso en el inicio de salitas de jardín o primaria, así como dificultades de aprendizaje y/o conducta, sin 
que esto fuera interpretado como una relación de tipo causal.

Pudimos confirmar algún tipo de problemática en el ámbito escolar -aprendizaje y/o conducta- en el $48 \%$ de los chicos detectados como maltratados en la muestra, aumentando en un $6 \%$ respecto al relevamiento anterior. No se informa sobre problemas en el $25 \%$. Pero resulta llamativo el $27 \%$ de los casos en donde la respuesta es que lo ignora. Salvo en aquellos casos en que se ha hecho cargo una maestra suplente -en cuyo caso al no conocer a su grado o sección, se trató que contestara la cartilla otra autoridad o maestra especial-se presenta como preocupante la falta de seguimiento de los alumnos bajo su responsabilidad.

Por último se indagó especialmente acerca de la situación de aquellos niños que presentan dificultades en su escolarización, res-pecto a la atención recibida o sea si reciben algún tipo de orientación terapéutica o de apoyo de algún tipo.

Esta información resulta comple-mentaria a la presentada anteriormente respecto a la repitencia o atraso de los alumnos. El $55 \%$ de los chicos que presentan dificultades está bajo alguna forma de tratamiento -216 sobre 393 chicos-.. Al mismo tiempo frente a los mismos chicos que son detectados como maltratados por una parte, y con problemas de repitencia o atraso por el otro, se ignora en el $20 \%$ de los mismos si reciben algún tipo de tratamiento. El $25 \%$ no está recibiendo ningún abordaje.

\section{DISCUSIÓN}

A partir del relevamiento realizado es necesario puntualizar algunas cuestiones:

1. La ocurrencia de los malos tratos familiares y entre pares se observa en todo el ámbito capitalino, los datos obtenidos para cada distrito deben ser tomados en forma orientativa y relativizarse, sobre todo en aquellos que devolvieron las cartillas sin responder. La falta de respuesta no es sinónimo de ausencia de casos. Es por ello que el menor número de casos de un distrito respecto de otro, podría solamente estar indicando una subdetección por diversos motivos.

2. Debemos señalar, también, la diferencia entre los datos obtenidos por nuestro relevamiento y aquellos con los que cuentan los equipos de orientación escolar de cada distrito, en muchos casos los nuestros los superaban -había casos que no habían llegado- y otros en que no nos llegaban a nosotros. Algo similar ocurre si comparamos los casos detectados respecto a maltrato físico y abuso sexual infantil y los que atienden en los servicios especializados de salud y justicia, que superan am-pliamente a los mencionados en este reevamiento.

3. La situación anterior se relaciona directamente con las dificultades encontradas en el desarrollo del relevamiento. Pese al acuerdo previo y al apoyo e interés de la Secretaría de Educación, la tarea fue dificultosa. Se plantearon reuniones previas con cada supervisor de nivel inicial y primaria -que previamente habían sido contactados por la Secretaria de Educación-, para explicar los objetivos de la investigación y pedir la colaboración de los mismos.

4. Posteriormente, éstos comuni-caban a los directores el inicio de la misma, recién en estos momentos la directora y/o la jefa de campo del proyecto se ponía en contacto con cada autoridad, verificando su información, realizando las aclaraciones pertinentes y avisando el horario en que se visitaría la escuela. Pese a todos estos recaudos, muchas veces concurrían las profesionales para entregar las cartillas y no podían acceder al establecimiento porque se habían olvidado de comunicarlo a todas las autoridades.

Por otra parte, salvo excepciones, no se facilitó un espacio adecuado de encuentro con los docentes para explicar suficientemente las car-tillas previo a su llenado. Estas situaciones también fueron señaladas como obstaculizadoras por los profesionales que llevaron a cabo este tipo de relevamiento en las comunidades españolas, por lo que se trata de ciertas resistencias normales a este tipo de indagación.

5. En cuanto a las dificultades concretas encontradas, observamos en muchos ámbitos cierto desinterés y evitación con el relevamiento poniendo en duda su aplicabilidad, y que a partir de estos datos realmente se planificara desde la Secretaría algún tipo de acción. En varias ocasiones se temía que el registrar casos diera una mala imagen de la escuela; otra excusa muy frecuente era las diversas ocupaciones que tenían "como para agregar algo más".

Esto debe ser analizado cuidadosamente, para tratar de entender la actual situación, que se encuadra en momentos coyunturales muy difíciles: las docentes se encuentran recargadas de trabajo y al mismo tiempo, imposibilitadas de incorporar aspectos informativos $u$ orientativos que alivien su pesada carga cotidiana -como en 
el caso del abordaje del maltrato infantil-.

6. Asimismo, cuando se observa la presencia del maltrato, suele adjudicarse el problema casi exclusivamente a la familia, hemos visto muchas situaciones de malos tratos en la misma escuela, que se encuadrarían en el denominado maltrato institucional. Este era observable, a varios niveles, en las autoridades y docentes frente a los chicos y frente a nuestro equipo; en las autoridades frente a los docentes y nosotras; en las docentes entre sí. Fueron mencionados en un $3 \%$ dentro de "otras formas" como la violencia institucional, pese a que no estaba incluida esta forma en la tipología de la cartilla.

Hemos observado, también un gran desconocimiento respecto al uso de instrumentos para evaluar situaciones familiares y de malos tratos y desconfianza frente a la aplicación de cuestionarios, lo que llevó muchas veces a resistencias frente a nuestra tarea.

7. Asimismo, debemos señalar que el número de casos detectados en la muestra relevada asciende a 1590 y corresponde al $7 \%$ del total de instituciones educativas dependientes de la Secretaría de Educación -nivel inicial y primario- y al $38 \%$ de los distritos escolares. Podemos esperar, por lo tanto, la existencia de un número mucho más alto de niños que estén sufriendo alguna forma de malos tratos infantiles en el total de escuelas y distritos.

\section{CONCLUSIONES}

Finalmente, al resumir las principales conclusiones del relevamiento de casos en los niveles inicial y primario, queremos detenernos en la relación existente en la ocurrencia de los malos tratos y su impacto según la etapa evolutiva del niño. Debemos tener en cuenta que las habilidades parentales cambian según la edad, necesidades y capacidad de los niños (Cerezo, 1996). Es en esta instancia donde se registra la importancia de desarrollar tareas de prevención del maltrato infantil, sobre todo en el período que abarca desde el nacimiento hasta los 5 años de vida. Las guarderías maternales y las escuelas de nivel inicial, se manejan con un grupo etáreo de máximo riesgo. Deben estar capacitados para detectar señales de riesgo o de malos tratos efectivos, al mismo tiempo que resulta sumamente prioritario el desarrollo de charlas a los padres advirtiendo sobre la fragilidad de los niños y los riesgos que corren en su desarrollo cuando los padres no están mínimamente adiestrados para su función.

Así mismo, es importante la capacitación en la detección temprana de los casos, es por ello que consideramos que existiendo varios instrumentos estandarizados para su uso en el campo educativo y/o sanitario, los mismos deberían ser incorporados en las etapas diag-

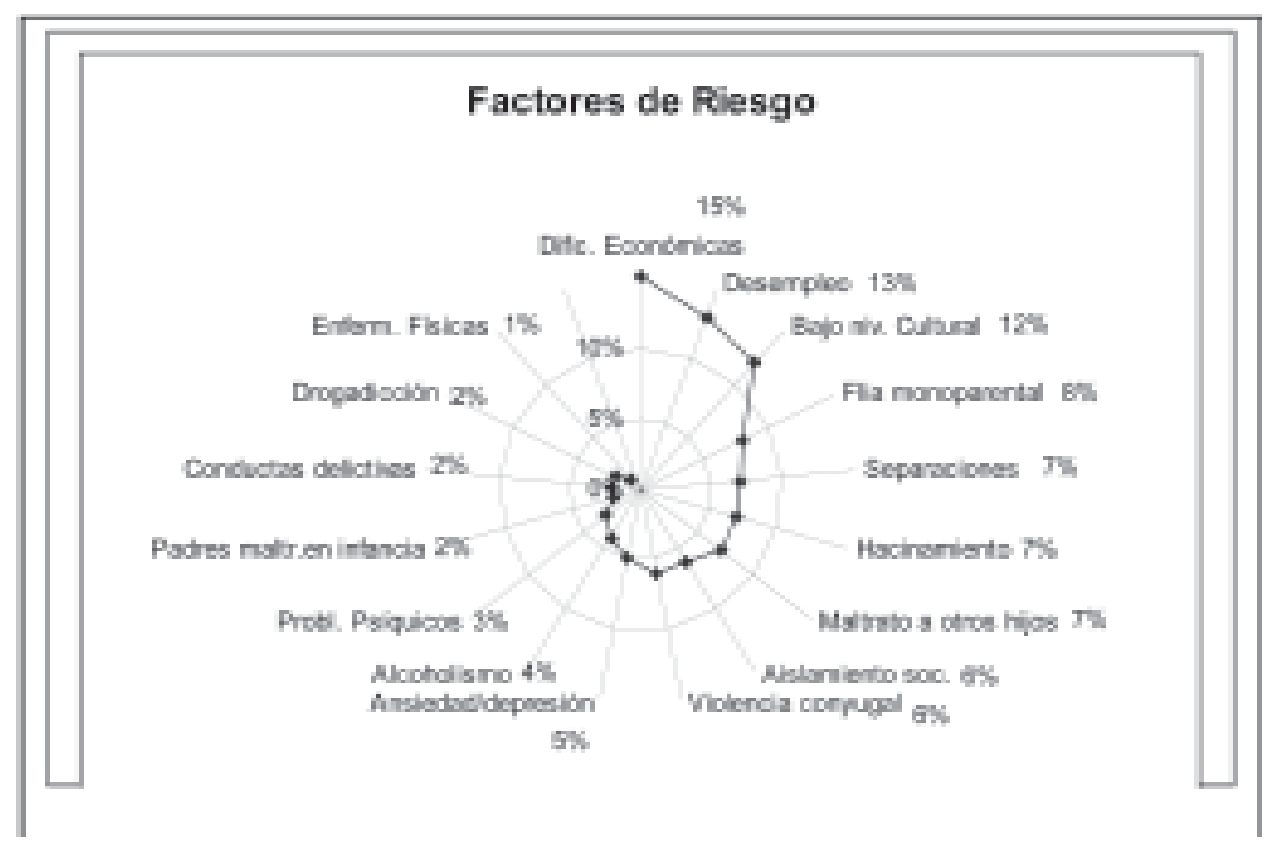

CUADRO 2. FACTORES DE RIESGO 
nósticas y posteriormente en la evaluación de los abordajes terapéuticos realizados, ya que brindan la posibilidad de lograr resultados más objetivos sobre la evaluación del caso.

En el contexto de crisis de nuestros países, las políticas de satisfacción de las necesidades básicas desplazan, en el mejor de los casos, a los programas de prevención y abordaje de la violencia familiar y el maltrato infantil. Los cambios mencionados en los roles genéricos y en la estructura familiar, la desocupación, la violencia urbana y la doméstica, la inadecuación de las respuestas institucionales y de las políticas públicas y sociales, llevan a un replanteo profundo de lo realizado hasta ahora y de los pasos a seguir. Se observa la existencia de situaciones "nuevas" que implican la revisión de marcos teórico/conceptuales y de estrategias de abordaje para conocer e intervenir en la problemática de la violencia familiar $y$, concretamente, en los niños.

Frente a esta situación consideramos que es fundamental actuar sobre todo en prevención, tanto en el ámbito familiar como en el escolar. Las charlas, la información que se brinde y sobre todo la realización de talleres de reflexión y participación, sólo muy lentamente podrán ir revirtiendo estas situaciones. El registro elevado de situaciones de violencia entre pares y entre grupo requiere de acciones planificadas dentro de la escuela que involucren a todos los sectores-personal de conducción, docentes, padres y alumnos-. Reconocemos las dificultades que implica encararlo de esta manera, hemos trabajado en una investigación posterior -UBACYT 2001/2003- en varias escuelas, y a pesar de lo difícil que resulta un abordaje múltiple prácticamente es el único adecuado para lograr modificaciones a mediano plazo.

Debemos señalar también, como urgente, el análisis del maltrato institucional en sus diferentes formas y la victimización secundaria que el mismo produce en el ámbito sanitario, educativo y jurídico. En estos momentos reducir la problemática del maltrato infantil al espacio intrafamiliar resulta reduccionista; la compleja situación que estamos atravesando muestra dificultades en varios niveles. Está surgiendo un conjunto de problemas, muchos de los cuales nos plantean el desafío de ser nuevos, derivados de todo lo anteriormente mencionado y que tienen un fuerte impacto en la crianza de los hijos y en la satisfacción de sus necesidades, que provocan un alto nivel de estrés y dificultades en el acceso a la educación, la salud y la vivienda... ya que todos estos factores mencionados son reconocidos a nivel mundial como de alto riesgo para las disfunciones familiares y por lo tanto para la posible aparición de la violencia en el seno familiar.

Hoy, esto no afecta sólo a los tradicionales sectores populares, que se ven acrecentados con los nuevos pobres o indigentes, abarca más allá: a los sectores medios que han caído en la escala social y a los que luchan por mantenerse. Frente a estos problemas, no podemos dejar de mencionar el papel que deberían jugar las políticas públicas y sociales, que resultan imprescindibles para el desarrollo de estrategias de intervención que permitan desarticular el "circuito de la crisis» y por lo tanto en muchos casos el «circuito de la violencia». Varios programas de tratamiento y recuperación de familias violentas llevados a cabo en otros países garantizan un subsidio durante el período en que transcurre el tratamiento, además de realizar un tiempo prudencial de seguimiento a fin de asegurar su inserción laboral y social. De ninguna manera se inter-pretan como suficientes las distintas técnicas psicoterapéuticas especializadas en el tema, sin una adecuada inserción social de estas familias. La inserción implica no sólo lo laboral, si-no las redes de apoyo social y los vínculos que fortalezcan la recuperación.

Queremos señalar, por último la importancia que las condiciones cotidianas de vida, hoy en Argentina, tienen sobre el fenómeno de la "violencia", especialmente en la conformación de las subjetividades y en las concepciones que se van construyendo acerca del desempeño de los roles parentales, los cambios en la maternidad y la paternidad, los estilos de crianza, lo que se considera desarrollo "normal" del niño, la tolerancia al estrés, fantasías y opciones en el fracaso de las expectativas de crecimiento en este contexto y en los modelos a transmitir a los hijo/as y las nuevas formas de familia, entre otros.

REFERENCIAS

Abad, S. (2001). Violencia en las instituciones. En R. Teubal (Comp.), Violenciafamiliar, trabajo social e instituciones (pp. 133-157). Buenos Aires: Paidós.

Aries, P. (1987). El niño y la familia en el Antiguo Régimen. Madrid: Taurus.

Averbuj, G., Bozzalla, L., Marina, M., Tarantino, G. \& Zaritzky, G. (Comps.). (2005). Violencia y escuela: 
propuestas para comprender y actuar. Buenos Aires: Aique.

Badinter, E. (1991). ¿Existe el amor maternal? Historia del amor maternal: siglos XVII al XX. Madrid: Paidós.

Barudy Labrin, J. (1998). El dolor invisible de la infancia: una lectura ecosistémica del maltrato infantil. Barcelona: Paidós,

Barudy Labrin, J. \& Marquebreucq, A.-P. (2006). Hijas e hijos de madres resilientes. Barcelona: Gedisa.

Belsky, J. (1993). Etiology of child maltreatment: a developmental-ecological analysis. Psychological Bulletin, 114(3), 413-34.

Bringiotti, M. I. (1998, marzo). El embarazo adolescente como factor de riesgo para el maltrato y abandono infantil. Revista de la Federación de Asociaciones para la Prevención del Maltrato Infantil, 4(1), 65-74.

Bringiotti M. I. (1998). Violencia social y maltrato infantil en Argentina. Implicancias de los cambios ocurridos en los últimos años en su crecimiento y desarrollo. En I. Izaguirre (Comp.), Violencia social y derechos humanos (pp. 165-175). Buenos Aires: EUDEBA.

Bringiotti, M. I. (1999). Maltrato infantil: factores de riesgo para el maltrato físico. Madrid: Miño y Dávila.

Bringiotti, M. I. (2000). La escuela ante los niños maltratados. Buenos Aires: Paidós.

Bringiotti, M. I. (en prensa). La violencia cotidiana en el ámbito escolar. Algunas posibles propuestas de prevención y abordaje. Buenos Aires: Lugar editorial.

Bringiotti, M. I., Krynveniuk, M. \& Lassi, S. (2005). Las múltiples violencias de la "violencia" en la escuela. Hacia un abordaje integrativo del problema. En G. Averbuj, L. Bozzalla, M. Marina, G. Tarantino, \& G. Zaritzky (Comps.), Violencia y escuela (pp. 25-47). Buenos Aires: Aique.

Bringiotti, M. I. \& Palazzo, S. A. (2007). Palabras y espejos. Aportes para la prevención de la transmisión de la violencia familiar. Buenos Aires: Lumen.

Bringiotti, M. I. \& Samaniego, C. (2003, diciembre). La crisis socioestructural Argentina y su impacto en la infancia. Bienestar y Protección Infantil, 2(1), 79-91.

Burguière A., Klapisch-Zuber C., Segalen M. \& Zonabend, F. (1988). Historia de la familia. Madrid: Alianza.

Cantón Duarte, J. \& Cortés Arboleda, R. (1997). Malos tratos y abuso sexual infantil: causas, consecuencias e intervención. Madrid: Siglo XXI.

Cerezo Ramírez, F. (1999). Conductas agresivas en edad escolar. Madrid: Pirámide.

Cordié, A. (1998). Malestar en el docente. Buenos
Aires: Nueva Visión.

De Mause, L. (Ed.). (1974). Historia de la infancia. Madrid: Alianza Universidad.

Finkelhor, D. \& Baron, L. (1986). Risk factors for child sexual abuse. Journal of Interpersonal Violence, 1(1), 43-71.

González Tornaría, M. L., Vandemeulebroecke, L. \& Colpin, H. (2001). Pedagogía familiar. Montevideo: Trilce.

Gracia Fuster, E. \& Musitu Ochoa, G. (1993). El maltrato infantil. Un análisis ecológico de los factores de riesgo. Madrid: España. Ministerio de Asuntos Sociales.

Imberti, J. (Comp.). (2001). Violencia y escuela. Miradas y propuestas concretas. Buenos Aires: Paidós.

Intebi, I. (Comp.) (2006). Maltrato de niños, niñas y adolescentes. Investigación epidemiológica. Población infantil de Sarmiento, provincia de Chubut. Buenos Aires: Familias del Nuevo Siglo.

Lapetra Coderque, P. (1994). La comunidad escolar y el maltrato infantil. En: Primera Jornadas sobre Infancia Maltratada: el Maltrato Institucional (pp. 45-63). Alava, España: Asociación Vasca para la Ayuda a la Infancia Maltratada.

López, F.; Etxebarría, I.; Fuentes, M. J. \& Ortiz, M. J. (1999). Desarrollo afectivo y social. Madrid: Pirámide.

Matias, A. da Silva Araujo. (2004). Estudo da prevalência de maustratos em crianças de 0 a 6 anos matriculadas em creches e préescolas da rede pública e particular na cidade de Ribeirão Preto$S P$. Tesis de maestría no publicada, Universidade de São Paulo, Faculdade de Filosofia, Ciências e Letras de Ribeirão Preto.

Paúl Ochotorena, J. de (1990). Cartilla epidemiológica. Comunicación per-sonal.

Paúl Ochotorena, J. de \& Arruabarrena M. I. (1986). Manual de protección infantil. Barcelona: Masson.

Redondo, P. (2004). Escuelas y pobrezas. Entre el desasosiego y la obstinación. Buenos Aires: Paidós.

Voor, W. (2005). Bullying. El acoso escolar. Barcelona: Ediciones Oniro.

Wainerman, C. (2005). La vida cotidiana en las nuevas familias. ¿Una revolución estancada? Buenos Aires: Lumiere. 